\title{
Medication-related osteonecrosis of the jaw - a disease with many names
}

Sven Otto ${ }^{1}$

1 Ludwig-Maximilians-Universität München

\begin{abstract}
Since the first description of medication induced necrosis of the jaw in 2003 by Marx, much has been written and published about this disease. In the following, we will examine this disease and examine its development - on the one hand which definitions exist, why they are defined differently and how they are classified.
\end{abstract}

\section{Definitions}

Definition of bisphosphonate-related osteonecrosis of the jaw in 2007

Defined by American Association of Oral and Maxillofacial Surg eons Advis ory Task Force on Bis phosphonateRelated Ostenonecrosis of the Jaws

Definition of bisphosphonate-related osteonecrosis of the jaw in 2009

Defined by Salvatore Ruggiero et al.

Definition of medication-related osteonecrosis of the jaw in 2014

Defined by Salvatore L. Ruggiero et al.

As a result of demog raphic change, older and polymorbid patients are more and more in (dental) medical practices and clinics. As a result, prescriptions of antiresorptive drugs such as denosumab or bisphosphonates are on the rise. Bisphosphonates and antiresorptive drugs such as denosumab have their particular benefit in treatment of osteoporosis $^{[1]}$ and treatment of skeletal-metastatic and underlying oncological diseases ${ }^{[2]}$.

A rare but difficult to treat complication of this antiresorptive treatment is Medicationrelated osteonecrosis of the jaw MRONJ, short ONJ. Medication-related osteonecrosis of jaw are characterized by exposed necrotic bone in the maxillofacial region. Other common symptoms include pain, soft tissue swelling, intra- or extraoral fistula formation or abscessing or impairment to the nerve function. MRONJ is more common in the mandible (around $2 / 3$ of the cases) and less common in the maxilla (around 1/3 of the cases). In both jaws the molar and premolar regions are affected more frequentlyy ${ }^{[3][4]}$. 
The exact pathogenesis of medication-related osteonecrosis of jaw is still unclear. Some authors assume that a combination of low bone turnover with local characteristics of the oral cavity, in particular mechanical stress, coupled with bacterial infection and frequently occurring germ entry ports are the likely causes for MRON ${ }^{[5]}$. In several large case series and studies, it has been shown that mostly patients with malignant underlying diseases, especially breast cancer, multiple myeloma and prostate cancer, suffer from medication-related osteonecrosis of jaw, while the risk in treatment of osteoporosis is significantly lower. This is due to the dosages and applied application intervals (cumulative dose), because bioavailability is being significantly higher, especially of bisphosphonates, when administered intravenously than when taken orally. Furthermore, tobacco smoking and diabetes mellitus may increase the risk of developing medication-related osteonecrosis of jaw ${ }^{[2][6]}$.

Bisphosphonates are in medical use since the late 1960ies ${ }^{[7]}$. Over time bisphosphonates haven been developed further and the introduction of nitrogen-containing bisphosphonates was certainly a breakthrough regarding the potency of the drugs. In this respect the use became more and more widespread in the treatment of osteoporosis and skeletal involvement of malignant diseases. In 2003 the first scientific reports emerged dealing with a hitherto unknown disease which was described initially as an avascular necrosis of the jaw bone under pamidronate and zoledronate use. The term "bisphosphonate-associated osteonecrosis of the jaw" was mentioned only in the ensuing discussion ${ }^{[8]}$ but was used further on by many authors.

\section{$\underline{2007}$}

Over the years, more and more patient cases were published. The American Association of Oral and Maxillofacial Surgeons (AAOMS) defining the "bisphosphonate-associated osteonecrosis of the jaw" for the first time in 2007 . Here BRONJ was defined as follows ${ }^{[9]}$ :

Definition of bisphosphonate-related osteonecrosis of the jaw in 2007

In addition, a classification was established:

- Stage 1: Exposed/necrotic bone in patients who are asymptomatic and have no evidence of infection

- Stage 2: Exposed/necrotic bone associated with infection as evidenced by pain and erythema in region of exposed bone with or without purulent drainage

- Stage 3: Exposed/necrotic bone in patients with pain, infection, and one or more of the following: pathologic fracture, extraoral fistula, or osteolysis extending to the inferior border 
$\underline{2009}$

In 2009, a revised version of the BRONJ definition was published in a position paper by the AAOMS $^{[10]}$ :

Definition of bisphosphonate-related osteonecrosis of the jaw in 2009

The classification was adjusted as follows:

- Stage 0: No clinical evidence of necrotic bone, but nonspecific clinical findings and symptoms

- Stage 1: Exposed and necrotic bone in asymptomatic patients without evidence of infection

- Stage 2: Exposed and necrotic bone associated with infection as evidenced by pain and erythema in region of exposed bone with or without purulent drainage

- Stage 3: Exposed and necrotic bone in patients with pain, infection, and one or more of the following: exposed and necrotic bone extending beyond the region of alveolar bone, (ie, inferior border and ramus in the mandible, maxillary sinus and zygoma in the maxilla) resulting in pathologic fracture, extraoral fistula, oral antral/oral nasal communication, or osteolysis extending to the inferior border of the mandible or the sinus floor

Except for the fact that the AAOMS removed the term "necrotic" from the 2007 BRONJ definition and introduced "Stage 0", in 2009, unlike the definition of 2007, nothing has changed.

\section{$\underline{2014}$}

According to the current definition and the most recent position paper of 2014 of the AAOMS, the following definition was made ${ }^{[11]}$ :

\section{Definition of medication-related osteonecrosis of the jaw in 2014}

Also the classification has been changed minimally:

- Stage 0: No clinical evidence of necrotic bone, but non-specific clinical findings, radiographic changes and symptoms

- Stage 1: Exposed and necrotic bone, or fistulae that probes to bone, in patients who are asymptomatic and have no evidence of infection

- Stage 2: Exposed and necrotic bone, or fistulae that probes to bone, associated with infection as evidenced by pain and erythema in the region of the exposed bone with or without purulent drainage

- Stage 3: Exposed and necrotic bone or a fistula that probes to bone in patients with pain, infection, and one or more of the following: exposed and necrotic bone 
extending beyond the region of alveolar bone,(i.e., inferior border and ramus in the mandible, maxillary sinus and zygoma in the maxilla) resulting in pathologic fracture, extra-oral fistula, oral antral/oral nasal communication, or osteolysis extending to the inferior border of the mandible of sinus floor

The important change of the position paper in 2014 concerns the former description of "exposed necrotic bone". This description has been changed to "exposed bone or bone that can be examined by an intraoral or extraoral fistula." However until now osteonecrosis of the jaw remains not a histologically confirmed term. The definition and respectively the diagnosis relies on one clinical finding (bone exposure) and anamnestic information (intake of antiresorptive or antiangiogenic drugs and absence of irradiation to the jaw area). Antiangiogenic drugs were included in the definition for the first time, so that not only patients with antiresorptive drugs (bisphosphonates and denosumab), but also those using antiangiogenic drugs such as bevacizumab are now covered by the definition.

\section{Nomenclature}

Over the time, more and more new nomenclatures have been discussed. Initially, the "bisphosphonate related osteonecrosis of jaw" (BRONJ) was described ${ }^{[8]}$. In addition, "bisphosphonate-induced osteonecrosis of the jaw (BIONJ)"[12] and "bisphosphonateassociated osteonecrosis of the jaw (BAONJ)" were discussed ${ }^{[13]}$. In turn, other authors accented the infectious genesis and used the term "bisphosphonate-associated osteomyelitis of the jaw (BAOMJ)"10.4158/EP.14.9.1150[14] or "bisphosphonate-related osteomyelitis of the jaw (BROMJ)" ${ }^{[15]}$.

Further it became clear that not only bisphosphonates are the main risk factor to cause an osteonecrosis of the jaw, but also an intake of other antiresorptives such as denosumab ${ }^{[16]}$, so that the term "antiresorptive drug-related osteonecrosis of the jaw (ARONJ)" was established.

Other authors emphasize the different pharmacodynamics of these drugs and therefore use the term "denosumab-related osteonecrosis of the jaw, DRONJ" to differentiate the condition of BRON][17][18].

In 2014, the AAOMS also changed the name "bisphosphonate-related osteonecrosis of the jaw (BRONJ)" to "medication-related osteonecrosis of the jaw" (MRONJ) in its position paper. Thus, the potential risk of osteonecrosis was seen not only in association with bisphosphonates but also in other antiresorptive and antiang iog enic drugs ${ }^{[11]}$. 


\section{Conclusion}

There are many definitions for the medication-related osteonecrosis of jaw (MRONJ). However, each one of these terms reflects the current state and limitations of research. This shows that the topic of osteonecrosis of the jaw is still in development.

\section{References}

1. ^ Robert E. Marx. (1983). Osteoradionecrosis: A new concept of its pathophysiology.J Oral Maxillofac Surg, vol. 41

2. a, b Cornelia Then, Natalie Hörauf, Sven Otto, Christoph Pautke, Emmo von Tresckow, Tim Röhnisch. (2012). Incidence and Risk Factors of Bisphosphonate-Related Osteonecrosis of the Jaw in Multiple Myeloma Patients Having Undergone Autologous Stem Cell Transplantation. Onkologie, vol. 35 (11), 658-664.

3. ^ Sven Otto, Christian Schreyer, Sigurd Hafner, Gerson Mast, Michael Ehrenfeld, Stephen Stürzenbaum. (2012). Bisphosphonate-related osteonecrosis of the jaws Characteristics, risk factors, clinical features, localization and impact on oncological treatment. Journal of Cranio-Maxillofacial Surgery, vol. 40 (4), 303-309.

4. ^ Suad Aljohani, Robert Gaudin, Julian Weiser, Matthias Tröltzsch, Michael Ehrenfeld, Gabriele Kaeppler. (2018). Osteonecrosis of the jaw in patients treated with denosumab: A multicenter case series. Journal of Cranio-Maxillofacial Surgery, vol. 46 (9), 1515-1525.

5. ^ Robert E. Marx, R.P. Johnson. (1987). Studies in the radiobiology of osteoradionecrosis and their clinical significance. Oral Surg Oral Med Oral Pathol, vol. 64(4)

6. ^ (2015). Medication-Related Osteonecrosis of the Jaws.

7. ^ H. Fleisch, R.G. Russell, F. Straumann. (1966). Effect of pyrophosphate on hydroxyapatite and its implications in calcium homeostasis.. Nature, vol. 212(5065)

8. a, b Robert E. Marx. (2003). Pamidronate (Aredia) and zoledronate (Zometa) induced avascular necrosis of the jaws: a growing epidemic.. J Oral Maxillofac Surg, vol. 61(9)

9. ^ (2007). American Association of Oral and Maxillofacial Surgeons Position Paper on Bisphosphonate-Related Osteonecrosis of the Jaws. Journal of Oral and Maxillofacial Surgery, vol. 65 (3), 369-376.

10. ^ Salvatore L. Ruggiero, Thomas B. Dodson, Leon A. Assael, Regina Landesberg, Robert E. Marx, Bhoomi Mehrotra. (2009). American Association of Oral and Maxillofacial Surgeons Position Paper on Bisphosphonate-Related Osteonecrosis of the Jaws-2009 Update. Journal of Oral and Maxillofacial Surgery, vol. 67 (5), 2-12. 
11. a, b Salvatore L. Ruggiero, Thomas B. Dodson, John Fantasia, Reginald Goodday, Tara Aghaloo, Bhoomi Mehrotra. (2014). American Association of Oral and Maxillofacial Surgeons Position Paper on Medication-Related Osteonecrosis of the Jaw-2014 Update. Journal of Oral and Maxillofacial Surgery, vol. 72 (10), 1938-1956.

12. ^ Robert E. Marx. (2008). Bisphosphonate-induced osteonecrosis of the jaws: a challenge, a responsibility, and an opportunity. Int J Periodontics Restorative Dent, vol. 28(1)

13. ^Aliya A. Khan, George K.B. Sandor, Edward Dore, Archibald D. Morrison et al.. (2008). Canadian consensus practice guidelines for bisphosphonate associated osteonecrosis of the jaw.. J Rheumatol, vol. 35(7)

14. `Sunil Wimalawansa. (2008). Bisphosphonate-Associated Osteomyelitis of the Jaw: Guidelines for Practicing Clinicians. Endocrine Practice, vol. 14 (9), 1150-1168.

15. ^ Toru Yamazaki, KatsuTakahashi, Kazuhisa Bessho. (2014). Recent Clinical Evidence in Bisphosphonate-related Osteomyelitis of the Jaw: Focus on Risk, Prevention and Treatment. Rev Recent Clin Trials, vol. 9(1)

16. ^ John W. Hellstein, Robert A. Adler, Beatrice Edwards, Peter L. Jacobsen, John R. Kalmar, Sreenivas Koka. (2011). Managing the care of patients receiving antiresorptive therapy for prevention and treatment of osteoporosis. The Journal of the American DentalAssociation, vol. 142 (11), 1243-1251.

17. ^ Oliver Ristow, Carlos Gerngroß, Markus Schwaiger, Bettina Hohlweg-Majert, Victoria Kehl, Heike Jansen. (2014). Effect of antiresorptive drugs on bony turnover in the jaw: denosumab compared with bisphosphonates. British Journal of Oral and Maxillofacial Surgery, vol. 52 (4), 308-313.

18. ^ Kenneth E. Fleisher, Risto Kontio, Sven Otto. Antiresorptive Drug-related Osteonecrosis of the Jaw (ARONJ) - a Guide to Research. 